\section{Commentary: How to say goodbye}

\author{
Chethan P. Venkatasubba Rao, MD, ${ }^{\mathrm{a}}$ and \\ Subhasis Chatterjee, $\mathrm{MD}^{\mathrm{b}, \mathrm{c}}$
}

Neurologic complications during extracorporeal membrane oxygenation (ECMO) can be devastating. In this issue of the Journal, Migdady and colleagues ${ }^{1}$ provide valuable insight into the vexing problem of determining brain death (BD) in patients on ECMO, which we increasingly encounter with the expanding use of ECMO for cardiopulmonary resuscitation (CPR) and other salvage applications. The authors systematically review 22 studies (with 177 patients) of BD determination when conventional apnea testing (AT) cannot sufficiently confirm BD, which is the case in about $20 \%$ of patients supported by ECMO. These studies are summarized in descriptive tables to allow meaningful comparisons between them.

According to the Extracorporeal Life Support Organization (ELSO) data from 2015 to 2019, the prevalence of BD and overall neurologic complications in patients receiving ECMO ranges from $1.2 \%$ and $4.7 \%$ in respiratory failure to $1.7 \%$ and $7.3 \%$ in adult cardiogenic shock to $6.2 \%$ and $16.4 \%$ in CPR. ${ }^{2}$ This represents a significant reduction compared with an earlier ELSO analysis of patients who received venoarterial-ECMO from 1992 to 2013, which revealed an incidence of $7.9 \%$ for BD and $15.1 \%$ for neurologic complications. ${ }^{3}$ This downward trend over the past decade may reflect improved patient selection, management, and experience. Nevertheless, neurologic complications probably remain underestimated, because diagnostic imaging may be inconsistently used across centers, participation in registries is voluntary, and reporting of complications is not standardized.

From the a Department of Neurology, Section of Vascular Neurology and Neurocritical Care and ${ }^{\mathrm{b}}$ Michael E. DeBakey Department of Surgery, Divisions of General and Cardiothoracic Surgery, Baylor College Medicine; and ${ }^{\mathrm{c}}$ Department of Cardiovascular Surgery, Texas Heart Institute, Houston, Tex.

Disclosures: The authors reported no conflicts of interest.

The Journal policy requires editors and reviewers to disclose conflicts of interest and to decline handling or reviewing manuscripts for which they may have a conflict of interest. The editors and reviewers of this article have no conflicts of interest.

Received for publication April 13, 2020; revisions received April 13, 2020; accepted for publication April 13, 2020; available ahead of print May 5, 2020.

Address for reprints: Subhasis Chatterjee, MD, Baylor College of Medicine, One Baylor Plaza, MS: BCM 390, Houston, TX 77030-3411 (E-mail: subhasis. chatterjee@bcm.edu).

J Thorac Cardiovasc Surg 2021;162:878-9

$0022-5223 / \$ 36.00$

Copyright (c) 2020 by The American Association for Thoracic Surgery

https://doi.org/10.1016/j.jtcvs.2020.04.112

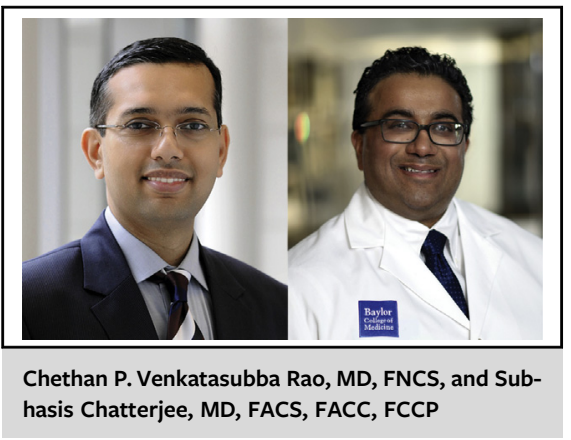

CENTRAL MESSAGE

During ECMO, apnea testing to

determine brain death can be a

challenge to conduct and diffi-

cult to interpret. Determining

brain death reliably often re-

quires confirmatory testing.

BD is defined as irreversible coma with an identified cause, unresponsiveness, absence of movement, breathing, and brainstem reflexes. ${ }^{4}$ Cultural and religious differences in the acceptance of BD must be sensitively addressed by clinicians. In patients with devastating, irreversible neurologic injury, a thorough neurologic examination to assess brainstem reflexes, interpretation of computed tomographic imaging, and exclusion of confounding factors (medications, laboratory abnormalities, hypotension, hypothermia, hypoxia) are the basis of the BD criteria established by the American Academy of Neurology. ${ }^{5}$ In addition, formal AT is performed to assess brainstem function by demonstrating the absence of a respiratory drive after 8 to $10 \mathrm{mi}-$ nutes of hypercapnia (arterial carbon dioxide tension $>60 \mathrm{~mm} \mathrm{Hg}$, or $20 \mathrm{~mm} \mathrm{Hg}$ above baseline values). ${ }^{6} \mathrm{As}$ the authors delineate, there is no consensus method of AT in standard practice; therefore, unsurprisingly, BD testing in patients receiving ECMO varies widely. ${ }^{7}$ Indeed, only $50 \%$ of patients in this review actually underwent AT. Avoiding hemodynamic instability and maintaining adequate oxygenation during the period of hypercapnia can be challenging, which may explain why AT was performed in only one half of the brain-dead patients. Stopping the sweep gas flow and discontinuing ventilation runs the risk of hypoxemia, while simply adding carbon dioxide into the ventilator or ECMO gas mixture can have variable results.

Although some countries require a second, confirmatory BD test, in the United States it is dictated by state law and is 
sometimes left to the treating physician's discretion. Indeed, although $77 \%$ of patients in this review had confirmatory AT, only $12 \%$ were declared BD by AT alone. Largely, clinicians wanted more confirmation, so $84 \%$ of patients who had confirmatory AT had at least 1 additional test. The tests most commonly used include electroencephalography (most used), computed tomographic or cerebral angiography, transcranial Doppler ultrasonography, and technetium nuclear imaging. Nevertheless, changes in oxygenation, ventilation, and blood flow patterns in patients receiving ECMO have not been rigorously analyzed, nor have their effects on the results of various confirmatory tests. Guidance is needed from major societies (eg, American Academy of Neurology, ELSO) regarding how to best perform AT in patients receiving ECMO and the use of confirmatory tests.

Direct conversations with family are important-ideally before cannulation (which may not be feasible); otherwise, soon after ECMO initiation-regarding a time limit for ECMO. In our practice, especially in the ECMO-for-CPR setting, where the risk of hypoxic brain injury is greatest, we suggest families consider discontinuing ECMO for futility if no meaningful neurologic recovery occurs within 5 to 7 days or if the patient is still dependent on ECMO without meaningful cardiac recovery or bridging options after 7 to 10 days. Ethics and palliative care teams can be valuable resources, and close collaboration with neurology colleagues is necessary to avoid conflicting messages. Most patients with persistent coma or severe anoxic encephalopathy typically do not progress to formal BD determination but rather undergo palliative withdrawal of life support when meaningful recovery becomes very unlikely.

Finally, BD in patients receiving ECMO offers the opportunity for organ-preserving ECMO. In Spain in 2016, 18\% of all deceased donations were controlled donations with organ-preserving ECMO after circulatory death. ${ }^{8}$ With the shortage of donors, ethical donation is feasible and should be considered. ${ }^{9}$ Certainly, the ethical use of ECMO in these situations-organ harvest as a consequence versus the goal—requires thoughtful deliberation. ${ }^{10}$

This systematic review provides a thoughtful survey of $\mathrm{BD}$ practices in patients receiving ECMO and underscores the lack of standardization of neurologic evaluation in determining BD. It also raises important questions: What is optimal neurologic monitoring in patients receiving ECMO? When and in whom do we initiate such monitoring? What should trigger BD examination, and when? How do we standardize monitoring and BD determination? Finally, how can we educate the families of patients receiving ECMO about $\mathrm{BD}$, and will such education improve organ procurement or resource use? This is a call for our professional organizations to develop a consensus set of guidelines.

\section{References}

1. Migdady I, Stephens RS, Price C, Geocadin RG, Whitman G, Cho SM. The use of apnea test and brain death determination in patients on extracorporeal membrane oxygenation: a systematic review. J Thorac Cardiovasc Surg. 2021;162: 867-77.e1.

2. Extracorporeal Life Support Organization. ECLS Registry report. Available at: https://www.elso.org/Registry/Statistics/InternationalSummary.aspx. Accessed April 9, 2020.

3. Lorusso R, Barili F, Mauro MD, Gelsomino S, Parise O, Rycus PT, et al. In-hospital neurologic complications in adult patients undergoing venoarterial extracorporeal membrane oxygenation: results from the Extracorporeal Life Support Organization registry. Crit Care Med. 2016;44:e964-72.

4. Uniform Determination of Death Act, 12 Uniform Laws Annotated (U.L.A.) 589 (West 1993 and West Supp. 1997).

5. Wijdicks EF, Varelas PN, Gronseth GS, Greer DM. Evidence-based guideline update: determining brain death in adults: report of the Quality Standards Subcommittee of the American Academy of Neurology. Neurology. 2010;74:1911-8.

6. Wijdicks EF. The diagnosis of brain death. N Engl J Med. 2001;344:1215-21.

7. Lie SA, Hwang NC. Challenges of brain death and apnea testing in adult patients on extracorporeal membrane oxygenation-a review. J Cardiothorac Vasc Anesth. 2019;33:2266-72.

8. Perez-Villares JM, Lara-Rosales R, Fernandez-Carmona A, Fuentes-Garcia P, Burgos-Fuentes M, Baquedano-Fernandez B. Mobile ECMO team for controlled donation after circulatory death. Am J Transplant. 2018;18:1293-4.

9. Dalle Ave AL, Gardiner D, Shaw DM. The ethics of extracorporeal membrane oxygenation in brain-dead potential organ donors. Transpl Int. 2016;29:612-8.

10. Migliaccio ML, Zagli G, Cianchi G, Lazzeri C, Bonizzoli M, Cecchi A, et al. Extracorporeal membrane oxygenation in brain-death organ and tissues donors: a single-centre experience. Br J Anaesth. 2013;111:673-4. 